\title{
CARTAS GEOTÉCNICAS: DESENVOLVIMENTO DE SOFTWARE GERADOR DE PERFIL DE RESISTÊNCIA À PENETRAÇÃO DO SUBSOLO DO UNASP-EC
}

\author{
Clayson Albino do Nascimento \\ Centro Universitário Adventista de São Paulo, Bacharel em Engenharia Civil \\ Engenheiro Coelho, SP, Brasil \\ clayson.albino@hotmail.com \\ Mario Roberto Barraza Larios \\ Centro Universitário Adventista de São Paulo, Doutor em Engenharia Civil \\ Engenheiro Coelho, SP, Brasil \\ mario.larios@unasp.edu.br
}

\begin{abstract}
RESUMO
Em vista do tempo decorrente da execução de investigações de subsolo, custos e outros inconvenientes, este artigo propõe a utilização de uma biblioteca de perfis de sondagens para a criação de uma série de cartas geotécnicas por meio de uma aplicação digital que foi desenvolvida. Esta aplicação foi alimentada com dados da resistência à penetração do subsolo dentro da área de estudo do Centro Universitário Adventista de São Paulo Campus Engenheiro Coelho. Portanto, a consulta ao perfil vertical do subsolo passa a ocorrer de maneira simples e rápida, onde o software exibe os números de golpes registrados no Standard Penetration Test (SPT) por camadas e o nível da água subterrânea em dados estatisticamente confiáveis, facilitando o projeto de fundações para novas edificações.
\end{abstract}

Palavras-chave: Investigação de subsolo. Geoestatística. Standard Penetration Test (SPT). Software. Carta Geotécnica.

\section{GEOTECHNICAL CHARTS: DEVELOPMENT OF SOFTWARE GENERATOR OF RESISTANCE PROFILE TO SUBSOIL PENETRATION. DEVELOPED AND TESTED PROJECT INTO UNASP-EC}

\begin{abstract}
In view of the time taken to carry out underground investigations, costs and other inconveniences, this paper proposes the use of a library of sounding profiles for the creation of a series of geotechnical charts using a novel digital application. This application was fed with data of penetration resistance of the subsoil from the studied area that is the Centro Universitário Adventista de São Paulo - Campus Engenheiro Coelho. Therefore, the query to the vertical profile of the subsoil is made in a simple and fast way, as the software shows the values of strokes recorded in the Standard Penetration Test (SPT) layered and the groundwater level in statistically reliable data, facilitating the foundations project for new buildings.
\end{abstract}

Keywords: Subsoil investigation. Geostatistics. Standard Penetration Test (SPT). Software. Geotechnical Chart.

\section{INTRODUÇÃO}

Dentre os diversos tipos de ensaios de campo utilizados na área da engenharia civil, destaca-se como um dos mais comuns e, talvez, o mais utilizado por conta da acessibilidade, o denominado Standard Penetration Test, representado pela sigla SPT. Por meio deste, é possível apontar algumas informações cruciais para projetos de fundações, estabilidade de taludes, estruturas de contenção, análises de risco, entre outros (REBELLO, 2008). Tais elementos envolvem as condições naturais do solo no local sondado, as suas características físicas, a resistência à penetração $\left(N_{S P T}\right)$ e a determinação da profundidade do nível da água. Todas estas informações são comumente apresentadas através do perfil de sondagem,

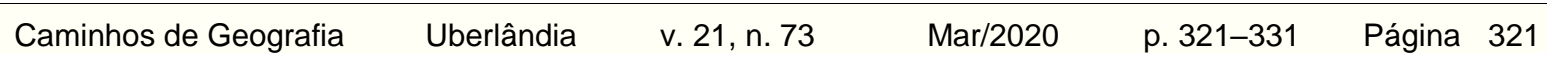


gerado após os devidos exames das amostras coletadas no ensaio, de acordo com os itens 1 e 7.2 da Norma Brasileira (NBR) 6484 (ABNT, 2001).

Contudo, como afirmou Miranda (2008), o perfil de sondagem possui visão do exato ponto onde ocorreu o ensaio, havendo uma possível chance de que o subsolo localizado na proximidade possua comportamento divergente. Pensando exatamente nas variações que podem ocorrer na estrutura do subsolo, Daniel C. Krige fez uso das chamadas médias móveis para aproximar dados geológicos sem resultar em superestimações (ANDRIOTTI, 2004).

Através dos estudos de Krige publicados em 1951, desenvolveu-se o método geoestatístico chamado de Krigagem, o qual faz uso de todas as amostras de ensaios numa determinada área para, estatisticamente, prever um perfil em qualquer ponto em derredor (ANDRIOTTI, 2004). A utilização deste método, discretizado em uma complexa rotina de cálculos por Silva Júnior (2006) e uma biblioteca de perfis de sondagens à percussão realizadas no Centro Universitário Adventista de São Paulo, organizadas pelas autoras Barbosa e Ávila (2013), tornou possível a geração de uma série de cartas geotécnicas, que será objeto de estudo para a presente pesquisa.

As chamadas cartas geotécnicas podem fornecer diversos tipos de informações ambientais (FREITAS e CAMPANHA, 2007). Entretanto, neste caso, as cartas utilizadas apresentam somente a interpolação da resistência do solo em forma de números de golpes no ensaio de SPT (NSPT). Estas cartas encontravamse separadas em arquivos distintos, classificados por metro de profundidade e eram acessadas de forma totalmente manual, o que representa suscetibilidade a erros por descuido, ignorância, informação enganosa e outros fatores aleatórios, como afirma El-Ramly (2001, apud ZUCCOLO, 2016).

Portanto, tendo em vista a intenção de aprimorar o tempo de serviço das equipes de engenharia em projetos de fundações, a ferramenta computacional se faz capaz de ler todas as cartas geotécnicas de forma simultânea e organizada. A aplicação localiza as cartas sobre um mapa georreferenciado, a fim de gerar, com um único clique, o perfil vertical de resistência do solo à penetração (representados em NSPT). Complementando o produto da aplicação, gerou-se uma carta com a correlação dos variados níveis da água subterrânea, a qual também terá parte no perfil vertical. Tais objetivos visam facilitar os serviços preliminares e de estruturas de fundações para qualquer edificação que possa vir a ser iniciada na parte central do campus.

Assim, de acordo com Santos (2017), o programa possibilitará a estimação rápida de propriedades geotécnicas locais, podendo ser utilizado tanto para avaliações, quanto para concepções, possivelmente reduzindo a necessidade de realização de novos ensaios na área correlacionada. Consequentemente, os custos operacionais poderão ser reduzidos, juntamente com o tempo de espera das execuções de sondagens. Também possibilitará a melhor avaliação de dados e a expansão de correlações regionais. Sendo, portanto, uma ferramenta valiosa para o estudo de correlações e capacidade de carga na área da geotecnia, bem como alguns notáveis estudos dos últimos 50 anos, realizados por grandes pesquisadores como Velloso (1959), Schmertmann (1970), Martins e Miranda (2003), Souza et. al. (2012).

Com a intenção de que sejam acrescidas as informações de novos ensaios de sondagens no futuro, empregou-se uma forma de adicionar e posicionar novas cartas geotécnicas, possibilitando a contínua atualização da aplicação. A fim de guiar à utilização correta da ferramenta, é possível consultar o documento suplementar em forma de manual, anexado ${ }^{1}$ junto à pesquisa.

\section{CARTAS GEOTÉCNICAS: ANÁLISE ESTATÍSTICA DE NSPT}

Avaliar e prever o comportamento e a natureza de propriedades geotécnicas compreende uma tarefa complexa quando se pretende aplicar a estatística clássica. Sendo assim, para implementar a estatística espacial, leva-se em consideração um conceito clássico que sugere a utilização de um número

\footnotetext{
${ }_{1}^{1}$ Disponível em: https://1drv.ms/b/s!AtP-YqfRc8XEg4ogC0klH6ca3-uwXg?e=R0Cmr9
}

$\begin{array}{lllll}\text { Caminhos de Geografia Uberlândia } & \text { v. 21, n. } 73 & \text { Mar/2020 } & \text { p. 321-331 } & \text { Página } 322\end{array}$


significativo de amostras, a fim de colaborar para a obtenção de resultados próximos da realidade (STURARO, 1994 apud SOARES, 2011, p. 47).

A fim de que sejam realizadas as investigações em quantidade satisfatória, aconselha-se seguir às orientações de números mínimos de sondagens dispostas no item 4.1.1 da NBR 8036 (ABNT, 1983). Tal medida é indicada tendo em vista a carência de normatização dos parâmetros que envolvem o estudo para correlacionar a resistência do subsolo.

Os resultados da correlação podem ser avaliados através do coeficiente de determinação $R^{2}$. Este é responsável por medir o valor da variabilidade encontrada entre as amostras, abrangendo dois eixos em sua verificação. Contudo, vale ressaltar que a credibilidade da interpolação não é dada somente pela verificação deste coeficiente (SOARES, 2011).

Maia (2003) afirmou que, seja qual for o tipo de análise, podem-se utilizar diversas medidas descritivas para representar as propriedades de variação, tendência e forma. Baseado neste critério, nota-se a baixa resistência à penetração registrada pelas sondagens utilizadas para a pesquisa, pois através de valores medianos de NsPT constatou-se que o solo é predominantemente mole, segundo a NBR 6484 (ABNT, 2001).

Entende-se como um conceito de análise de resultados que a confiabilidade pode ser facilmente comprometida caso haja grande variação entre as amostras estudadas, admitindo que estes dois padrões ocorrem de forma inversa (ZUCCOLO, 2016). Tal fato justifica a utilização de cartas correlacionadas para a área em questão, pois o banco de dados apresenta baixa variação da resistência à penetração entre camadas em grande parte do território verificado.

\section{METODOLOGIA}

Para o desenvolvimento da pesquisa e da aplicação, foi utilizado como campo de testes o Centro Universitário Adventista de São Paulo Campus de Engenheiro Coelho. Com isto, a ferramenta pode ser utilizada em qualquer lugar onde exista um banco de dados de sondagens. Sendo assim, foi necessário coletar os devidos dados e complementá-los com novos ensaios que ainda não haviam sido registrados, ordenando o material conforme as suas locações. Todo o banco de dados precisou passar por uma atualização. Toda a metodologia é explicitada nas subseções de Coleta de Dados e de Nível D'água e Clima Local.

\section{COLETA DE DADOS}

Como parte dos estudos de Barbosa e Ávila (2013), foram recolhidos todos os perfis de sondagens com SPT que já haviam sido realizadas no Centro Universitário Adventista de São Paulo até 2013, compondo uma biblioteca com 44 perfis, numa área de aproximadamente $0,6 \mathrm{~km}^{2}$, que englobam pontos sob os prédios já construídos. Cada um destes locais sondados foi sequencialmente numerado, de forma que pudesse ser utilizado na composição de cartas geotécnicas sob procedimento igualmente pontuado por Zuquette (1987), fazendo utilização de um artifício da geoestatística, denominado de krigagem. Tal procedimento segue a rotina de cálculo do método de Krige, dissertado por Silva Júnior (2006).

A mesma biblioteca de sondagens utilizada por Ávila e Barbosa em 2013 foi tomada como fonte de dados para esta pesquisa. No entanto, novos ensaios complementaram o banco de dados a partir de então, totalizando, atualmente, 50 perfis de sondagens. Todo o material foi cedido pelo Centro Universitário e pode ser encontrado de forma ordenada na sequência de estudo da Figura 1.

Com a adição de novos perfis à base de dados, cria-se a necessidade de atualização. Sendo assim, foram geradas novas cartas geotécnicas, mantendo o método geoestatístico de krigagem ordinária. Após 
desenvolvidas, foram identificadas em tons de calor (frios e quentes). Cada qual representa um metro de profundidade, chegando até a $27^{\underline{a}}$ camada.

Figura 1 - Locação enumerada da biblioteca de sondagens.

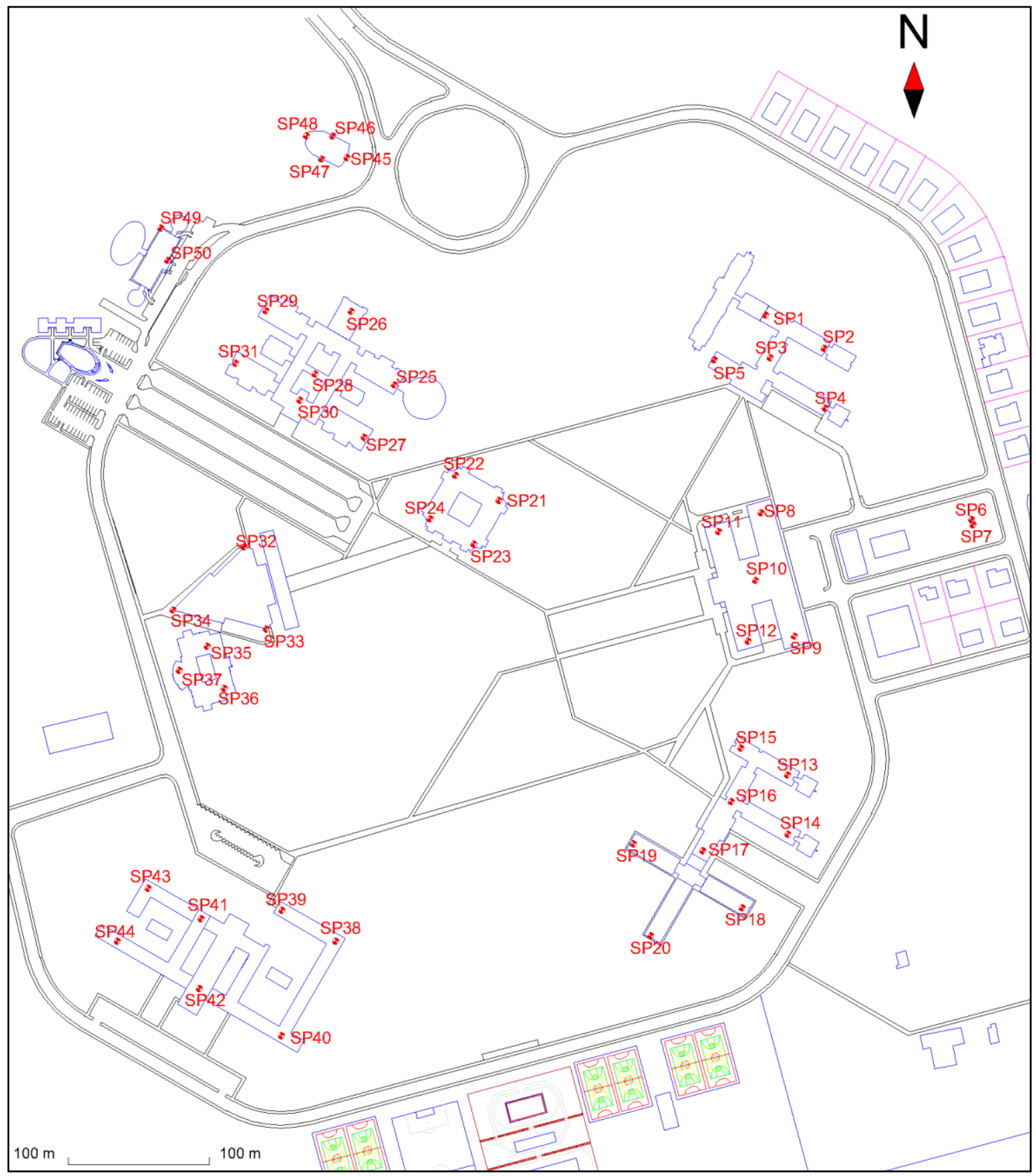

Fonte - AUTOR (2019)

\section{NÍVEL D'ÁGUA E CLIMA LOCAL}

De forma a colaborar com as concepções estruturais futuras, identificou-se o nível d'água de cada um dos pontos, correlacionando-os numa carta com valores expressos através de um marcador de gota azul e de valores expostos no topo do mapa vertical. No entanto, a profundidade interpolada da água subterrânea pode facilmente sofrer variações de acordo com a época do ano em que for comparada com resultados de campo, já que os ensaios foram realizados em datas distintas, variando entre abril, maio, outubro e dezembro. 
É possível notar a existência de períodos muito secos ou muito úmidos na região em estudo, o que pode acarretar um nível mais baixo ou mais alto da água subterrânea em função do mês de observação. Segundo o Climatempo (2019), a variação de precipitação no mês de janeiro pode chegar a ser dez vezes maior quando comparada ao mês de julho na cidade de Engenheiro Coelho, interior de São Paulo. Portanto, tendo em vista o efeito da sazonalidade, a carta de nível d'água pode apresentar alguma variação ao ser colacionada com a realidade. É possível observar na Figura 2 o gráfico contendo o traçado da chuva mensal média registrada nos últimos 30 anos.

Figura 2 - Chuva mensal média na cidade de Engenheiro Coelho-SP.

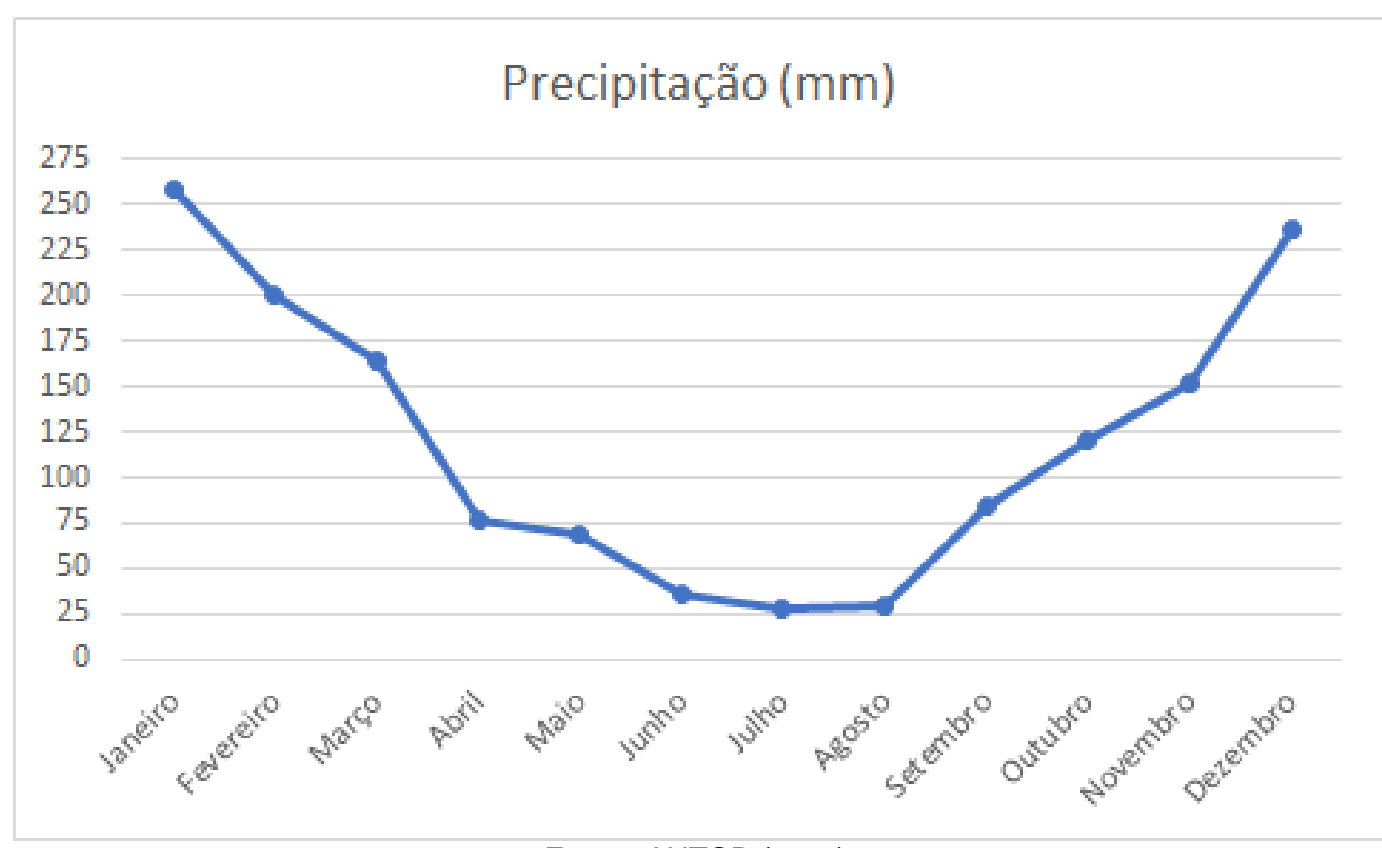

Fonte - AUTOR (2019).

\section{RESULTADOS}

O estudo dos resultados abrange desde a produção do software até a análise dos valores apresentados pelo mesmo. Tal material é discretizado e analisado em sua forma final nas subseções de Software Cartas Geotécnicas e Análise de Resultados.

\section{SOFTWARE CARTAS GEOTÉCNICAS}

Para a concepção funcional, utilizou-se o instrumento computacional desenvolvido pela Microsoft国, denominado Visual Studio Community 2017, que pode ser baixado e utilizado livremente como um ambiente de desenvolvimento integrado (IDE), capaz de gerar outras aplicações. Com interface bem intuitiva, o programa Cartas Geotécnicas possui como base interativa, a ferramenta aberta online Open Street Map $\AA$, o qual faz uso de dados geo-espaciais colaborativos, sob a licença Open Database License (OdbL) de 2018, para apontar o mapeamento e a georreferência em qualquer parte selecionada na área de estudo.

Tendo as cartas sido devidamente preparadas de acordo com o documento de manual em anexo ${ }^{2}$, a aplicação permite inseri-las de forma que permaneçam fixadas no local escolhido do mapa, como se estivessem exatamente uma abaixo da outra. Caso queira realizar uma consulta ao perfil vertical de resistência à penetração do subsolo, o usuário pode clicar onde exista a carta geotécnica ou introduzir as coordenadas no local adequado. Sendo assim, ao selecionar um ponto, o ícone de pino informará o local escolhido.

2 Disponível em: https://1drv.ms/b/s!AtP-YqfRc8XEg4ogC0klH6ca3-uwXg?e=R0Cmr9

$\begin{array}{lllll}\text { Caminhos de Geografia } & \text { Uberlândia } & \text { v. 21, n. } 73 & \text { Mar/2020 } & \text { p. 321-331 Página } 325\end{array}$


Ao realizar a seleção de algum ponto para consulta, estando localizado dentro da área demarcada, o programa faz a leitura instantânea de todas as cartas por ordem de profundidade. Ao ocorrerem os acessos, é registrado no painel de Mapa Vertical, à direita da tela, a cor referente à legenda escalar de resistência à penetração em cada metro de profundidade, juntamente com o NSPT propriamente dito. Através de um marcador azul, o painel aponta a profundidade do nível da água subterrânea.

A região de desenvolvimento do estudo apresenta baixa variabilidade na composição do subsolo, sendo predominantemente do tipo silte arenoso. Por este motivo, a primeira versão do software visa apresentar o perfil vertical do subsolo contendo informações somente do NSPT e do nível d'água subterrânea. No entanto, está em desenvolvimento o módulo interpolador do tipo de solo, o qual será implementado em versões futuras, facilitando ainda mais o dimensionamento de fundações para os usuários do programa.

Seguindo as orientações redigidas no documento suplementar, após a devida geração e inserção das cartas no software, o modelo criado no mesmo poderá ser acessado livremente. Logo na abertura do programa a interface se mostra intuitiva, apresentando as imagens das cartas sobre um mapa georreferenciado, juntamente com a escala de cores inserida nas cartas e o campo específico do mapa vertical.

Para consultar as cartas e gerar um mapa vertical pode-se clicar com o botão esquerdo do mouse em qualquer parte dentro da área correlacionada (1). Também funciona se o usuário digitar as coordenadas em latitude e longitude desejadas no local (2) e pressionar a tecla enter. Em seguida, o mapa surgirá no canto direito da tela (3), onde poderá ser visualizado o NSPT correlacionado em cada camada juntamente com a posição da água subterrânea, como exemplificado na Figura 3.

Figura 3 - Consulta ao Mapa Vertical.

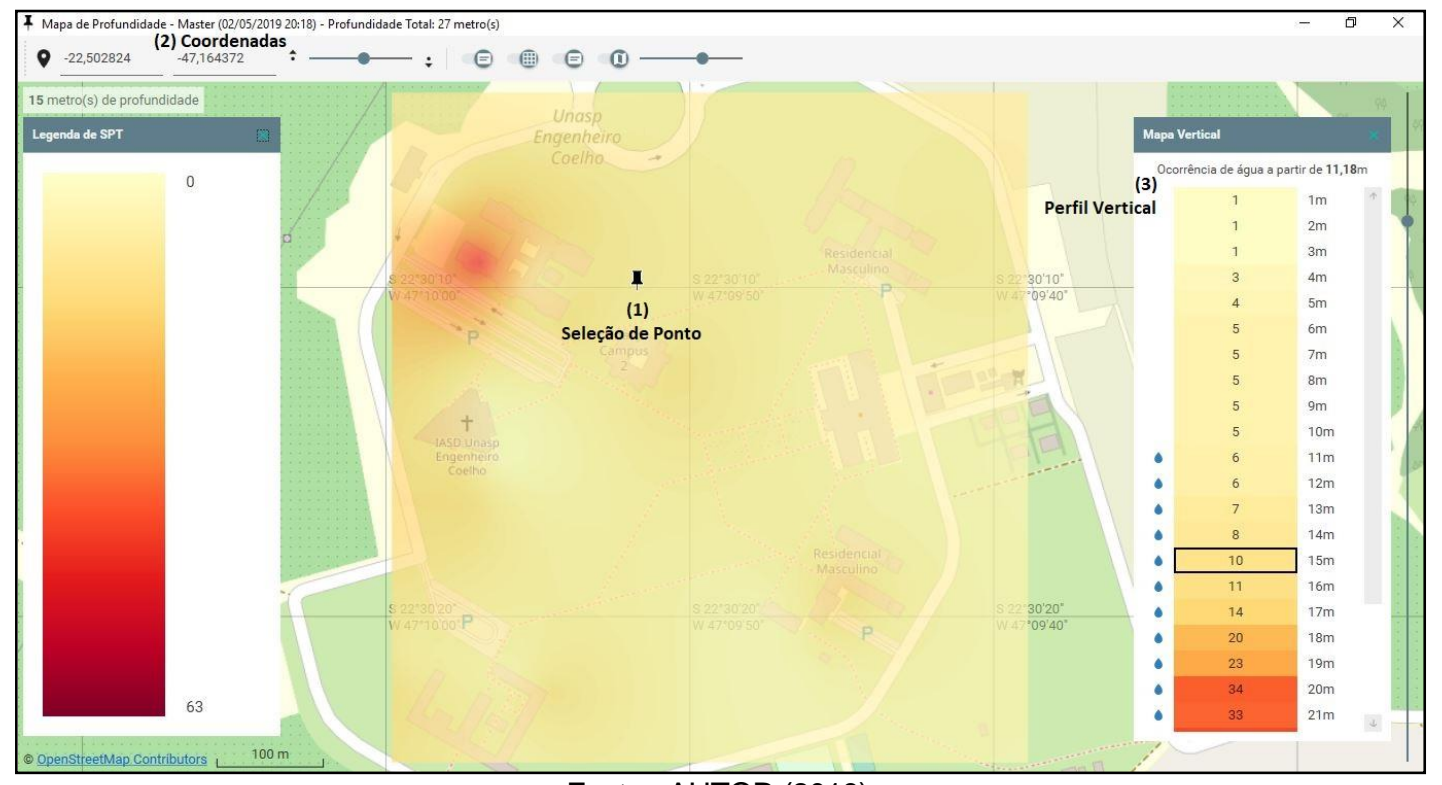

Fonte - AUTOR (2019).

\section{ANÁLISE DE RESULTADOS}

Admite-se que, dentre as muitas alternativas de análises, uma das mais adequadas é a de comparar os resultados apresentados pelo software com os perfis sondados no local. Utilizando o método de análise estatística denominado qui-quadrado $\left(\mathrm{X}^{2}\right)$, foram avaliadas as variações encontradas entre os dados do programa para os perfis interpolados e os valores reais sondados, que constam nos perfis de sondagens de todo o campus. Sendo assim, a validação da ferramenta computacional é feita utilizando a comparação dos dados do software e dos valores encontrados nas sondagens para as camadas de $3 \mathrm{~m}, 6 \mathrm{~m}, 10 \mathrm{~m}, 15 \mathrm{~m}$ e $25 \mathrm{~m}$ de profundidade.

Serão dispostos em tabelas os valores experimentais, os interpolados nos mapas e o desvio da relação entre eles. Para a análise do N $\mathrm{SPT}$ foram adotadas, então, seis camadas de profundidade, englobando toda a biblioteca de cartas. Os quadros contendo os valores de NSPT a serem analisados estão contidos nas Tabelas 1 e 2. 
Tabela 1 - Valores de NSPT para análise das camadas 3m, 6m e 10m.

\begin{tabular}{|c|c|c|c|c|c|c|c|c|c|}
\hline \multirow{2}{*}{$\begin{array}{c}\text { SPT } \\
\text { (Pontos) }\end{array}$} & \multicolumn{3}{|c|}{$3 m$} & \multicolumn{3}{|c|}{$6 m$} & \multicolumn{3}{|c|}{$10 \mathrm{~m}$} \\
\hline & REAL & MAPAS & $\begin{array}{c}\text { DESVIO } \\
(\%)\end{array}$ & REAL & MAPAS & $\begin{array}{c}\text { DESVIO } \\
(\%)\end{array}$ & REAL & MAPAS & $\begin{array}{c}\text { DESVIO } \\
(\%)\end{array}$ \\
\hline 1 & 6 & 4 & $-33,3$ & 8 & 7 & $-12,5$ & 9 & 7 & $-22,2$ \\
\hline 2 & 3 & 3 & 0,0 & 6 & 5 & $-16,7$ & 3 & 4 & 33,3 \\
\hline 3 & 5 & 4 & $-20,0$ & 8 & 7 & $-12,5$ & 8 & 7 & $-12,5$ \\
\hline 4 & 6 & 3 & $-50,0$ & 9 & 7 & $-22,2$ & 6 & 4 & $-33,3$ \\
\hline 5 & 3 & 3 & 0,0 & 7 & 5 & $-28,6$ & 8 & 7 & $-12,5$ \\
\hline 6 & 4 & 3 & $-25,0$ & 6 & 5 & $-16,7$ & 10 & 8 & $-20,0$ \\
\hline 7 & 4 & 3 & $-25,0$ & 9 & 5 & $-44,4$ & 7 & 8 & $\begin{array}{l}4,3 \\
\end{array}$ \\
\hline 8 & 2 & 2 & 0,0 & 5 & 4 & $-20,0$ & 5 & 4 & $-20,0$ \\
\hline 9 & 1 & 1 & 0,0 & 4 & 3 & $-25,0$ & 4 & 3 & $-25,0$ \\
\hline 10 & 2 & 1 & $-50,0$ & 6 & 4 & $-33,3$ & 6 & 4 & $-33,3$ \\
\hline 11 & 2 & 2 & 0,0 & 5 & 4 & $-20,0$ & 6 & 5 & $\begin{array}{l}-16,7 \\
\end{array}$ \\
\hline 12 & 2 & 1 & $-50,0$ & 6 & 4 & $-33,3$ & 8 & 7 & $\begin{array}{l}-12,5 \\
\end{array}$ \\
\hline 13 & 5 & 3 & $-40,0$ & 9 & 7 & $-22,2$ & 11 & 8 & $-27,3$ \\
\hline 14 & 3 & 3 & 0,0 & 4 & 4 & 0,0 & 11 & 10 & $-9,1$ \\
\hline 15 & 2 & 1 & $-50,0$ & 3 & 3 & 0,0 & 7 & 5 & $-28,6$ \\
\hline 16 & 4 & 3 & $-25,0$ & 7 & 4 & $-42,9$ & 11 & 8 & $-27,3$ \\
\hline 17 & 4 & 3 & $-25,0$ & 6 & 3 & $-50,0$ & 11 & 8 & $-27,3$ \\
\hline 18 & 3 & 3 & 0,0 & 5 & 4 & $-20,0$ & 8 & 8 & 0,0 \\
\hline 19 & 1 & 1 & 0,0 & 1 & 1 & 0,0 & 3 & 3 & 0,0 \\
\hline 20 & 2 & 2 & 0,0 & 4 & 3 & $-25,0$ & 5 & 4 & $-20,0$ \\
\hline 21 & 3 & 2 & $-33,3$ & 5 & 4 & $-20,0$ & 7 & 5 & $-28,6$ \\
\hline 22 & 2 & 2 & 0,0 & 6 & 4 & $-33,3$ & 5 & 3 & $-40,0$ \\
\hline 23 & 2 & 2 & 0,0 & 3 & 3 & 0,0 & 6 & 5 & $-16,7$ \\
\hline 24 & 3 & 2 & $-33,3$ & 5 & 4 & $-20,0$ & 7 & 4 & $-42,9$ \\
\hline 25 & 1 & 1 & 0,0 & 4 & 3 & $-25,0$ & 5 & 4 & $-20,0$ \\
\hline 26 & 2 & 2 & 0,0 & 4 & 3 & $-25,0$ & 6 & 4 & $-33,3$ \\
\hline 27 & 3 & 2 & $-33,3$ & 6 & 4 & $-33,3$ & 2 & 2 & 0,0 \\
\hline 28 & 2 & 2 & 0,0 & 3 & 3 & 0,0 & 5 & 3 & $-40,0$ \\
\hline 29 & 3 & 2 & $-33,3$ & 6 & 3 & $-50,0$ & 5 & 3 & $-40,0$ \\
\hline 30 & 2 & 2 & 0,0 & 6 & 3 & $-50,0$ & 3 & 3 & 0,0 \\
\hline 31 & 2 & 2 & 0,0 & 3 & 3 & 0,0 & 5 & 3 & $-40,0$ \\
\hline 32 & 1 & 1 & 0,0 & 2 & 2 & 0,0 & 3 & 3 & 0,0 \\
\hline 33 & 1 & 1 & 0,0 & 1 & 1 & 0,0 & 4 & 3 & $-25,0$ \\
\hline 34 & 1 & 1 & 0,0 & 1 & 1 & 0,0 & 4 & 3 & $-25,0$ \\
\hline 35 & 2 & 1 & $-50,0$ & 2 & 1 & $-50,0$ & 6 & 4 & $-33,3$ \\
\hline 36 & 2 & 1 & $-50,0$ & 4 & 2 & $-50,0$ & 5 & 6 & 20,0 \\
\hline 37 & 2 & 1 & $-50,0$ & 2 & 1 & $-50,0$ & 7 & 5 & $-28,6$ \\
\hline 38 & 5 & 4 & $-20,0$ & 4 & 4 & 0,0 & 4 & 4 & 0,0 \\
\hline 39 & 4 & 3 & $-25,0$ & 5 & 5 & 0,0 & 5 & 5 & 0,0 \\
\hline 40 & 2 & 1 & $-50,0$ & 5 & 5 & 0,0 & 5 & 4 & $-20,0$ \\
\hline 41 & 2 & 2 & 0,0 & 6 & 5 & $-16,7$ & 6 & 5 & $-16,7$ \\
\hline 42 & 1 & 1 & 0,0 & 5 & 5 & 0,0 & 5 & 4 & $-20,0$ \\
\hline 43 & 2 & 2 & 0,0 & 4 & 5 & 25,0 & 6 & 5 & $-16,7$ \\
\hline 44 & 4 & 2 & $-50,0$ & 6 & 5 & $-16,7$ & 5 & 5 & 0,0 \\
\hline 45 & 1 & 1 & 0,0 & 3 & 3 & 0,0 & 5 & 4 & $-20,0$ \\
\hline 46 & 1 & 1 & 0,0 & 3 & 2 & $-33,3$ & 4 & 4 & 0,0 \\
\hline 47 & 1 & 1 & 0,0 & 3 & 2 & $-33,3$ & 5 & 5 & 0,0 \\
\hline 48 & 2 & 1 & $-50,0$ & 2 & 2 & 0,0 & 5 & 5 & 0,0 \\
\hline 49 & 2 & 1 & $-50,0$ & 2 & 1 & $-50,0$ & 5 & 4 & $-20,0$ \\
\hline 50 & 2 & 1 & $-50,0$ & 2 & 1 & $-50,0$ & 4 & 4 & 0,0 \\
\hline
\end{tabular}


Tabela 2 - Valores de NsPT para análise das camadas 15m, 20m e 25m.

\begin{tabular}{|c|c|c|c|c|c|c|c|c|c|}
\hline \multirow{2}{*}{$\begin{array}{c}\text { SPT } \\
\text { (Pontos) }\end{array}$} & \multicolumn{3}{|c|}{$15 \mathrm{~m}$} & \multicolumn{3}{|c|}{$20 \mathrm{~m}$} & \multicolumn{3}{|c|}{$25 m$} \\
\hline & REAL & MAPAS & $\begin{array}{c}\text { DESVIO } \\
(\%)\end{array}$ & REAL & MAPAS & $\begin{array}{c}\text { DESVIO } \\
(\%)\end{array}$ & REAL & MAPAS & $\begin{array}{c}\text { DESVIO } \\
(\%)\end{array}$ \\
\hline 1 & 8 & 7 & $-12,5$ & 12 & 11 & $-8,3$ & 0 & 15 & 0,0 \\
\hline 2 & 11 & 8 & $-27,3$ & 13 & 11 & $-15,4$ & 0 & 13 & 0,0 \\
\hline 3 & 7 & 7 & 0,0 & 13 & 11 & $-15,4$ & 0 & 14 & 0,0 \\
\hline 4 & 9 & 8 & $-11,1$ & 8 & 9 & 12,5 & 10 & 10 & 0,0 \\
\hline 5 & 8 & 7 & $-12,5$ & 11 & 11 & 0,0 & 0 & 16 & 0,0 \\
\hline 6 & 8 & 7 & $-12,5$ & 22 & 18 & $-18,2$ & 46 & 40 & $-13,0$ \\
\hline 7 & 4 & 7 & 75,0 & 17 & 18 & 5,9 & 47 & 40 & $-14,9$ \\
\hline 8 & 7 & 7 & 0,0 & 14 & 11 & $-21,4$ & 42 & 29 & $-31,0$ \\
\hline 9 & 6 & 5 & $-16,7$ & 8 & 7 & $-12,5$ & 31 & 28 & $-9,7$ \\
\hline 10 & 6 & 5 & $-16,7$ & 11 & 9 & $-18,2$ & 0 & 24 & 0,0 \\
\hline 11 & 6 & 5 & $-16,7$ & 9 & 9 & 0,0 & 10 & 13 & 30,0 \\
\hline 12 & 7 & 5 & $-28,6$ & 10 & 9 & $-10,0$ & 0 & 25 & 0,0 \\
\hline 13 & 9 & 7 & $-22,2$ & 10 & 10 & 0,0 & 26 & 28 & 7,7 \\
\hline 14 & 9 & 8 & $-11,1$ & 12 & 11 & $-8,3$ & 38 & 33 & $-13,2$ \\
\hline 15 & 8 & 7 & $-12,5$ & 14 & 12 & $-14,3$ & 41 & 36 & $-12,2$ \\
\hline 16 & 15 & 11 & $-26,7$ & 22 & 17 & $-22,7$ & 37 & 36 & $-2,7$ \\
\hline 17 & 12 & 9 & $-25,0$ & 19 & 16 & $-15,8$ & 27 & 26 & $-3,7$ \\
\hline 18 & 7 & 7 & 0,0 & 8 & 11 & 37,5 & 9 & 17 & 88,9 \\
\hline 19 & 4 & 4 & 0,0 & 15 & 14 & $-6,7$ & 23 & 23 & 0,0 \\
\hline 20 & 4 & 3 & $-25,0$ & 9 & 10 & 11,1 & 24 & 20 & $-16,7$ \\
\hline 21 & 14 & 10 & $-28,6$ & 28 & 23 & $-17,9$ & 0 & 28 & 0,0 \\
\hline 22 & 8 & 8 & 0,0 & 23 & 26 & 13,0 & 0 & 29 & 0,0 \\
\hline 23 & 13 & 10 & $-23,1$ & 12 & 15 & 25,0 & 33 & 30 & $-9,1$ \\
\hline 24 & 10 & 8 & $-20,0$ & 18 & 20 & 11,1 & 0 & 31 & 0,0 \\
\hline 25 & 13 & 11 & $-15,4$ & 48 & 40 & $-16,7$ & 0 & 31 & 0,0 \\
\hline 26 & 16 & 15 & $-6,3$ & 25 & 22 & $-12,0$ & 0 & 33 & 0,0 \\
\hline 27 & 15 & 12 & $-20,0$ & 43 & 38 & $-11,6$ & 0 & 32 & 0,0 \\
\hline 28 & 10 & 15 & 50,0 & 40 & 31 & $-22,5$ & 0 & 33 & 0,0 \\
\hline 29 & 24 & 20 & $-16,7$ & 0 & 16 & 0,0 & 0 & 34 & 0,0 \\
\hline 30 & 12 & 18 & 50,0 & 0 & 32 & 0,0 & 0 & 33 & 0,0 \\
\hline 31 & 43 & 32 & $-25,6$ & 0 & 20 & 0,0 & 0 & 34 & 0,0 \\
\hline 32 & 5 & 7 & 40,0 & 16 & 17 & 6,3 & 0 & 33 & 0,0 \\
\hline 33 & 3 & 4 & 33,3 & 23 & 23 & 0,0 & 0 & 32 & 0,0 \\
\hline 34 & 13 & 13 & 0,0 & 36 & 33 & $-8,3$ & 0 & 0 & 0,0 \\
\hline 35 & 14 & 12 & $-14,3$ & 36 & 34 & $-5,6$ & 0 & 32 & 0,0 \\
\hline 36 & 16 & 14 & $-12,5$ & 32 & 37 & 15,6 & 0 & 32 & 0,0 \\
\hline 37 & 16 & 14 & $-12,5$ & 45 & 39 & $-13,3$ & 0 & 0 & 0,0 \\
\hline 38 & 14 & 10 & $-28,6$ & 19 & 15 & $-21,1$ & 34 & 31 & $-8,8$ \\
\hline 39 & 6 & 6 & 0,0 & 10 & 14 & 40,0 & 30 & 29 & $-3,3$ \\
\hline 40 & 7 & 7 & 0,0 & 15 & 17 & 13,3 & 43 & 39 & $-9,3$ \\
\hline 41 & 9 & 8 & $-11,1$ & 33 & 26 & $-21,2$ & 0 & 33 & 0,0 \\
\hline 42 & 11 & 10 & $-9,1$ & 31 & 26 & $-16,1$ & 0 & 36 & 0,0 \\
\hline 43 & 9 & 8 & $-11,1$ & 32 & 32 & 0,0 & 0 & 33 & 0,0 \\
\hline 44 & 15 & 11 & $-26,7$ & 53 & 37 & $-30,2$ & 0 & 36 & 0,0 \\
\hline 45 & 7 & 7 & 0,0 & 9 & 8 & $-11,1$ & 41 & 34 & $-17,1$ \\
\hline 46 & 9 & 7 & $-22,2$ & 9 & 8 & $-11,1$ & 32 & 31 & $-3,1$ \\
\hline 47 & 9 & 8 & $-11,1$ & 10 & 8 & $-20,0$ & 32 & 31 & $-3,1$ \\
\hline 48 & 8 & 7 & $-12,5$ & 9 & 8 & $-11,1$ & 36 & 33 & $-8,3$ \\
\hline 49 & 11 & 10 & $-9,1$ & 8 & 7 & $-12,5$ & 39 & 0 & $-100,0$ \\
\hline 50 & 9 & 10 & 11,1 & 9 & 7 & $-22,2$ & 37 & 0 & $-100,0$ \\
\hline
\end{tabular}

Da mesma forma, as medidas que são utilizadas para a análise do nível da água no subsolo são apresentadas na Tabela 3. 
Tabela 3 - Valores de níveis d'água para análise.

\begin{tabular}{|c|c|c|c|c|c|c|c|}
\hline $\begin{array}{c}\text { SPT } \\
\text { (Pontos) }\end{array}$ & $\begin{array}{c}\text { REAL } \\
(\mathrm{m})\end{array}$ & $\begin{array}{c}\text { MAPAS } \\
(\mathrm{m})\end{array}$ & $\begin{array}{c}\mathrm{DESVIO} \\
(\%)\end{array}$ & $\begin{array}{c}\mathbf{S P T} \\
(\mathbf{P o n t o s})\end{array}$ & $\begin{array}{c}\text { REAL } \\
(\mathrm{m})\end{array}$ & $\begin{array}{c}\text { MAPAS } \\
(\mathrm{m})\end{array}$ & $\begin{array}{c}\text { DESVIO } \\
(\%)\end{array}$ \\
\hline $\mathbf{1}$ & 14,60 & 13,52 & 7,40 & $\mathbf{2 6}$ & 13,02 & 11,87 & 8,83 \\
\hline $\mathbf{2}$ & 17,67 & 15,15 & 14,26 & $\mathbf{2 7}$ & 11,78 & 10,75 & 8,74 \\
\hline $\mathbf{3}$ & 14,00 & 13,15 & 6,07 & $\mathbf{2 8}$ & 13,28 & 12,13 & 8,66 \\
\hline $\mathbf{4}$ & 17,65 & 15,57 & 11,78 & $\mathbf{2 9}$ & 13,48 & 12,33 & 8,53 \\
\hline $\mathbf{5}$ & 14,30 & 12,71 & 11,12 & $\mathbf{3 0}$ & 12,73 & 11,68 & 8,25 \\
\hline $\mathbf{6}$ & 14,30 & 13,33 & 6,78 & $\mathbf{3 1}$ & 13,53 & 12,33 & 8,87 \\
\hline $\mathbf{7}$ & 14,53 & 13,15 & 9,50 & $\mathbf{3 2}$ & 11,01 & 10,15 & 7,81 \\
\hline $\mathbf{8}$ & 13,15 & 12,33 & 6,24 & $\mathbf{3 3}$ & 11,08 & 10,15 & 8,39 \\
\hline $\mathbf{9}$ & 14,02 & 12,71 & 9,34 & $\mathbf{3 4}$ & 11,12 & 10,15 & 8,72 \\
\hline $\mathbf{1 0}$ & 14,05 & 12,52 & 10,89 & $\mathbf{3 5}$ & 10,20 & 9,70 & 4,90 \\
\hline $\mathbf{1 1}$ & 12,92 & 11,68 & 9,60 & $\mathbf{3 6}$ & 11,40 & 9,48 & 16,84 \\
\hline $\mathbf{1 2}$ & 14,11 & 12,71 & 9,92 & $\mathbf{3 7}$ & 10,15 & 9,70 & 4,43 \\
\hline $\mathbf{1 3}$ & 13,10 & 12,13 & 7,40 & $\mathbf{3 8}$ & 9,32 & 8,83 & 5,26 \\
\hline $\mathbf{1 4}$ & 14,00 & 12,33 & 11,93 & $\mathbf{3 9}$ & 10,99 & 9,85 & 10,37 \\
\hline $\mathbf{1 5}$ & 15,90 & 13,70 & 13,84 & $\mathbf{4 0}$ & 11,75 & 10,52 & 10,47 \\
\hline $\mathbf{1 6}$ & 15,80 & 13,94 & 11,77 & $\mathbf{4 1}$ & 11,77 & 10,28 & 12,66 \\
\hline $\mathbf{1 7}$ & 16,40 & 14,12 & 13,90 & $\mathbf{4 2}$ & 11,74 & 10,52 & 10,39 \\
\hline $\mathbf{1 8}$ & 16,98 & 14,73 & 13,25 & $\mathbf{4 3}$ & 12,26 & 10,52 & 14,19 \\
\hline $\mathbf{1 9}$ & 17,45 & 15,15 & 13,18 & $\mathbf{4 4}$ & 12,70 & 11,00 & 13,39 \\
\hline $\mathbf{2 0}$ & 17,27 & 15,39 & 10,89 & $\mathbf{4 5}$ & 14,80 & 13,15 & 11,15 \\
\hline $\mathbf{2 1}$ & 11,97 & 11,00 & 8,10 & $\mathbf{4 6}$ & 14,83 & 13,33 & 10,11 \\
\hline $\mathbf{2 2}$ & 12,27 & 11,00 & 10,35 & $\mathbf{4 7}$ & 14,50 & 13,15 & 9,31 \\
\hline $\mathbf{2 3}$ & 12,48 & 11,00 & 11,86 & $\mathbf{4 8}$ & 14,95 & 13,33 & 10,84 \\
\hline $\mathbf{2 4}$ & 11,68 & 10,75 & 7,96 & $\mathbf{4 9}$ & 14,75 & 13,33 & 9,63 \\
\hline $\mathbf{2 5}$ & 11,92 & 11,00 & 7,72 & $\mathbf{5 0}$ & 14,65 & 13,15 & 10,24 \\
\hline
\end{tabular}

Fonte - AUTOR (2019)

Nota-se que os valores dos desvios-padrões são apresentados em percentuais, representando as taxas dos valores médios reais sondados. Pelo fato de os números registrados serem relativamente baixos, como por exemplo a camada de 3 metros, facilmente os desvios apresentam-se altos, ultrapassando a marca de $50 \%$. No entanto, servem como representação numérica de proporção.

Sempre que os desvios se apresentarem negativos, o mapa estará a favor da segurança, apontando medida inferior a real. De forma inversa, quando os desvios forem positivos, o mapa proporcionará número maior que o encontrado na sondagem. Caso o desvio seja igual a zero, significa que o mapa e o perfil de sondagem possuem o mesmo valor, como no SPT no 45 aos 15 metros, ou que o programa interpolou o NSPT no ponto mesmo quando sondagem naquele local foi interrompida em uma camada acima, como no SPT no 1 aos 25 metros. O desvio será de 100\% quando o programa não possuir valores suficientes para correlacionar tal área. Exemplo desta situação é encontrado aos 25 metros no furo de SPT de no 49 . O desvio resultará em $50 \%$ quando o valor real for o dobro da frequência esperada, como no SPT no 44 aos 3 metros.

A partir dos níveis da água e Nspt registrados nas Tabelas 1, 2 e 3 realizou-se o teste $X^{2}$, também chamado de Qui-Quadrado. Portanto, definem-se as colunas contendo os valores reais como sendo as frequências observadas, enquanto os valores correlacionados encontrados nas colunas dos mapas, referem-se às frequências esperadas. Deste modo, calcularam-se os chamados $p$-valor e $X^{2}$ calculado para cada teste, comparando-os com a porcentagem de significância e com o Xªbelado, respectivamente.

Há somente duas alternativas possíveis: aceitar ou rejeitar a hipótese nula, denominada H0. Caso esta condição seja aceita como verdadeira, indicará que não há diferença significativa entre as amostras, baseando-se no valor de significância $(\alpha)$. O nível de significância determinará o grau de precisão das amostras, sendo classificado como rigoroso quando adotado $\alpha=5 \%$ (STEINER et al., 2008). Por isso, este foi o nível de significância adotado nesta pesquisa. Os principais resultados do teste qui-quadrado podem ser encontrados na Tabela 4.

$\begin{array}{llllll}\text { Caminhos de Geografia } & \text { Uberlândia } & \text { v. 21, n. } 73 & \text { Mar/2020 } & \text { p. 321-331 } & \text { Página } 329\end{array}$


Tabela 4 - Valores resultantes do teste qui-quadrado $\left(\mathrm{X}^{2}\right)$.

\begin{tabular}{|c|c|c|c|c|c|c|}
\hline $\begin{array}{c}\text { Camadas de } \\
\text { Profundidade }\end{array}$ & P-Valor & $\begin{array}{c}\text { Grau de } \\
\text { Liberdade }\end{array}$ & $\boldsymbol{\alpha}$ & $\begin{array}{c}\mathbf{X}^{2} \text { Crítico } \\
\text { Tabelado }\end{array}$ & $\mathbf{X}^{2}$ Calculado & $\begin{array}{c}\text { Condição } \\
\text { Final }\end{array}$ \\
\hline $\mathbf{3 m}$ & 0,9998 & 49 & 0,05 & 66,3386 & 21,5000 & Aceitar H0 \\
\hline $\mathbf{6 m}$ & 0,9772 & 49 & 0,05 & 66,3386 & 31,2619 & Aceitar H0 \\
\hline $\mathbf{1 0 m}$ & 0,9994 & 49 & 0,05 & 66,3386 & 23,0500 & Aceitar H0 \\
\hline $\mathbf{1 5 m}$ & 0,9963 & 49 & 0,05 & 66,3386 & 26,5897 & Aceitar H0 \\
\hline $\mathbf{2 0 m}$ & 0,9652 & 46 & 0,05 & 62,8296 & 30,1974 & Aceitar H0 \\
\hline $\mathbf{2 5 m}$ & 0,6486 & 21 & 0,05 & 32,6706 & 18,0056 & Aceitar H0 \\
\hline $\begin{array}{c}\text { NíVEL } \\
\text { D'ÁGUA }\end{array}$ & 1,0000 & 49 & 0,05 & 66,3386 & 8,2680 & Aceitar H0 \\
\hline
\end{tabular}

Pode-se observar que os valores de $X^{2}$ calculado foram encontrados menores que os de $X^{2}$ tabelado. De igual forma, todos os testes apontaram o P-Valor maior que a taxa de significância. Portanto, a observação dos dois critérios diferentes conduz à invariável decisão: aceitar a hipótese nula, H0.

\section{CONSIDERAÇÕES FINAIS}

Atendendo às necessidades propostas pela pesquisa, o software Cartas Geotécnicas foi desenvolvido de forma a ser operado de maneira rápida e intuitiva, gerando de modo simples, o perfil vertical do subsolo. Em favor da veracidade dos dados, o teste estatístico de $\mathrm{X}^{2}$ apresentou resultados confiáveis, onde todas as camadas analisadas comprovam não haver diferenças significativas quando comparadas aos perfis de sondagens reais, pois em todas as situações testadas foram aceitas a hipótese nula $\mathrm{HO}$. Tal fato reforça a ideia de que, mesmo quando as amostras apresentaram desvio-padrão significativo, sua confiabilidade pode não ser estatisticamente afetada.

Sendo assim, o produto desta pesquisa facilitará a concepção de projetos de subestruturas para o Centro Universitário, pois reduzirá o tempo de estudo e os possíveis custos operacionais futuros, apresentando dados de procedência fidedigna para o serviço. No entanto, para que haja cada vez mais precisão e confiabilidade nos resultados apresentados, aconselha-se a atualizar constantemente as cartas, sempre que houver um novo perfil de sondagem disponível.

\section{REFERÊNCIAS}

ABNT - ASSOCIAÇÃO BRASILEIRA DE NORMAS TÉCNICAS. NBR 6484: Sondagens de Simples Reconhecimento com SPT - Método de Ensaio. Rio de Janeiro, ABNT, 2001.

NBR 8036: Programação de sondagens de simples reconhecimento dos solos para fundações de edifícios. Rio de Janeiro, ABNT, 1983.

ANDRIOTTI, J. L. Fundamentos da estatística e geoestatística. São Paulo: Unisinos, 2004.

BARBOSA, T. O.; ÁVILA, W. K. M. Carta Geotécnica para Investigações de Fundação do Centro Universitário Adventista de São Paulo Campus Engenheiro Coelho (UNASP-EC). 2013. Trabalho de Conclusão de Curso - Centro Universitário Adventista de São Paulo, Engenheiro Coelho, 2013.

CLIMATEMPO. ,Climatologia Engenheiro Coelho - SP.. São Paulo, 01 de abril de 2019. Disponível em: <https://www.climatempo.com.br/climatologia/2290/engenheirocoelho-sp $>$. Acesso em $01 \mathrm{de}$ abril de 2019.

EL-RAMLY, H. Probabilistic analyses of landslide hazards and risk: bridgning theory and practice. 2001, 391f. Tese (Doutorado) - Departament of Civil and Environmental Engineering, University of Alberta, Edmonton, Alta, 2001. 
FREITAS, C, G, L. de; CAMPANHA, V. A. Carta geotécnica e ambiental aplicada em planos diretores municipais. In: Simpósio Brasileiro de Cartografia Geotécnica e Geoambiental, 6, Uberlândia. Anais. Uberlândia: ABGE, 2007.

MAIA, J. A. C. Métodos probabilísticos aplicados à estabilidade de taludes e cavidade em rochas. 2003. Dissertação (Mestrado) - Universidade de Brasília, Brasília, 2003.

MARTINS, J.B., MIRANDA, T.F.S. Ensaios de Penetração nos Solos Graníticos da Região Norte de Portugal. Algumas Correlações. 2003. Revista Engenharia Civil. Departamento de Engenharia Civil - Universidade do Minho - Campus de Azurém. Portugal, 2003.

MIRANDA, T. C. Mapeamento das Unidades Geotécnicas e Desenvolvimento de um Sistema de Informações Geotécnicas para Área de Abrangência da Rodovia BR-101/RS. 2008. Dissertação (Pós-Graduação em Engenharia Civil) - Universidade Federal do Rio Grande do Sul, Rio Grande do Sul, 2008.

OPEN DEFINITION. Open Database License (ODbL) v1.0. Disponível em: $<$ https://opendatacommons.org/licenses/odbl/1.0/index.html>. Acesso em novembro de 2018.

REBELLO, Y. C. P. Fundações: Guia Prático de Projeto, Execução e Dimensionamento. 4ª Edição. São Paulo, SP. Zigurate Editora. 2008.

SANTOS, M. D. dos. Correlações entre sondagem de simples reconhecimento e resultados de ensaios de campo (SPT, CTP, DP) para diferentes subsolos arenosos. 2017. Dissertação (PósGraduação em Engenharia Civil) - Universidade Federal do Espírito Santo, Vitória, Espírito Santo, 2017.

SCHMERTMANN, J.H. Static cone to compute settlement over sand. Journal Soil Mechanics and Foundations Division, ASCE, v. 96, n. SM3, 1970.

SILVA JR, A. N. da. Uso da Krigagem para caracterização da variabilidade espacial da condutividade hidráulica no sem-árido nordestino com enfoque bayesiano. 2006. Dissertação (Pós-graduação) - Universidade Federal Rural de Pernambuco, Recife, 2006.

SOARES, W. C. Banco de dados geológico-geotécnicos com base em sondagens à percussão e uso de SIG: Análise espacial da profundidade do lençol freático e do NSPT para obras de fundação em João Pessoa - PB. 2011. Tese (Pós-Graduação em Geotecnia) - Universidade de São Carlos, São Paulo, 2011.

SOUZA, J.M.S., DANZIGER, B.R., DANZIGER, F.A.B. The Influence of the Relative Density of Sands in SPT and CPT Correlations. Soils and Rocks, São Paulo, v. 35, 2012.

STEINER, M. T. A., NETO, A. C., BRAULIO, A. N., ALVES, N. Métodos Estatísticos Multivariados Aplicados à Engenharia de Avaliações. 2008. Gestão \& Produção. Programa de Pós-Graduação em Métodos Numéricos em Engenharia - Universidade Federal do Paraná, Curitiba, Paraná, 2008. https://doi.org/10.1590/S0104-530X2008000100004

STURARO, J. R. Mapeamento Geoestatístico de Propriedades Geológico: Geotécnicas Obtidas de Sondagens de Simples Reconhecimento. Dissertação de Mestrado. Escola de Engenharia de São Carlos, Universidade de São Carlos. São Carlos. 1994.

VELLOSO, D. A. O Ensaio de Diepsondering e a Determinação da Capacidade de Carga do Solo. 1959. Rodovia, № 29. Rio de Janeiro, 1959.

ZUCCOLO, P. L. Procedimento para estimativa da confiabilidade geotécnica a partir de simulações numéricas: aplicação a um talude de solo não saturado. 2016. Dissertação (PósGraduação em Geotecnia) - Universidade de São Carlos, São Paulo, 2016.

ZUQUETTE, L. V. Análise crítica da cartografia geotécnica e proposta metodológica para condições brasileiras. 1987. Tese (Doutorado em Geotecnia) - Escola de Engenharia de São Carlos - Universidade de São Paulo, São Carlos, 1987.

Recebido em: 21/06/2019

Aceito para publicação em: 16/12/2019

Caminhos de Geografia Uberlândia v. 21, n. 73

Mar/2020

p. $321-331$

Página 331 